\title{
Hydrodynamics: Fluctuating initial conditions and two-particle correlations
}

\author{
R.P.G. Andrade, F. Grassi, Y. Hama *, W.-L. Qian ${ }^{1}$ \\ Instituto de Física, Universidade de São Paulo, Brazil \\ Received 30 July 2010; accepted 3 August 2010 \\ Available online 6 August 2010
}

\begin{abstract}
Event-by-event hydrodynamics (or hydrodynamics with fluctuating initial conditions) has been developed in the past few years. Here we discuss how it may help to understand the various structures observed in two-particle correlations.

(c) 2010 Elsevier B.V. All rights reserved.
\end{abstract}

Keywords: Hydrodynamic model; Fluctuating initial conditions; Ridge effect

\section{3+1 hydrodynamics: comparison with data}

One of the most striking results in relativistic heavy ion collisions is the existence of structures in the two-particle correlations [1-7] plotted as function of the pseudorapidity difference $\Delta \eta$ and the angular spacing $\Delta \phi$. The so-called ridge has a narrow $\Delta \phi$ located around zero and a long $\Delta \eta$ extent. The other structure located opposite has a single or double hump in $\Delta \phi$, its $\Delta \eta$ extent is not well established.

It has been suggested that the combined effect of longitudinal high-energy-density tubes (leftover from initial particle collisions) and transverse expansion is responsible for the ridge [8-12]. A hydrodynamics based explanation is attractive because of the various similarities between bulk matter and ridge (transverse momentum spectra, baryon/meson ratio, etc.). In order to compare with the ridge data, $3+1$ hydrodynamics must be used. Traditionally, smooth initial conditions

\footnotetext{
* Corresponding author.

E-mail address: hama@fma.if.usp.br (Y. Hama).

1 Now at the Instituto de Ciências Exatas, Universidade Federal de Ouro Preto, Ouro Preto - MG, Brazil.
} 


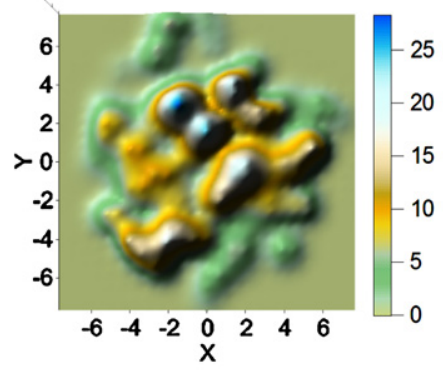

eta $=0$

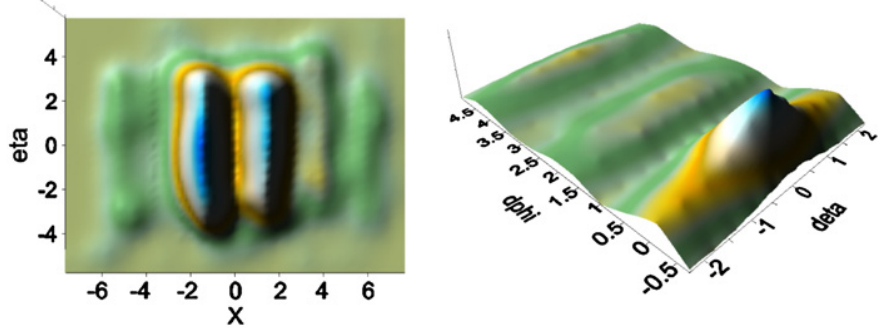

$Y=3.2$

Fig. 1. NeXSPheRIO central Au+Au collisions at $200 \mathrm{GeV}$ A: initial energy density and resulting two-particle correlation $\left(p_{t}^{\text {trig }}>2.5 \mathrm{GeV} \times p_{t}^{\text {assoc }}>1.5 \mathrm{GeV}\right)$.

have been used (see e.g. [13,14]). However some groups have started to develop an event-byevent approach: for each collision, some initial conditions are generated, hydrodynamics is run and results are stored. This is done many times thus mimicking experience. This method was pioneered by the Brazilian Collaboration SPheRIO [15-17] (some typical results can be seen in [18-21]). It has been used also by the German group with H. Petersen/M. Bleicher et al. [22]. More recently K. Werner and collaborators have also obtained results [23].

The SPheRIO group uses the NeXus code [24] to generate initial conditions and the Smoothed Particle Hydrodynamics method to solve the perfect fluid conservation equations. Results on twoparticle correlations can be found in $[21,25,26]$. H. Petersen/M. Bleicher et al. start with initial conditions generated with UrQMD and solve the perfect fluid hydrodynamics equations with a standard (grid) method. They have no results on two-particle correlations at RHIC. K. Werner et al. use EPOS initial conditions and also a standard (grid) method to solve the ideal hydrodynamics equations. Their results on two-particle correlations can be found in [23].

In the NeXSPheRIO approach, initial conditions have tubular structures and two-particle correlations exhibit near- and away-side ridges as can be seen in Fig. 1. As a double check, two different methods for elliptic flow subtraction have been used: ZYAM and event plane alignment. In the calculation by K. Werner et al., initial conditions also have tubular structures, a plane alignment method for elliptic flow subtraction is used and two-particle correlations exhibit near-side [23] and small away-side ridges [27].

In addition to reproducing both the near and away-side structures, NeXSPheRIO leads to good qualitative agreement with various data. For example, 1) for fixed $p_{t}^{\text {trig }}$ and increasing $p_{t}^{\text {assoc }}$, the near-side and away-side peaks decrease as seen in Fig. 2 for central collisions (this is generally expected since the number of associated particles decreases). On the other side, for fixed $p_{t}^{\text {assoc }}$ and increasing $p_{t}^{\text {trig }}$, the peaks increase. As shown below (see Fig. 5), this is because the singleparticle angular distribution becomes sharper, reducing the smearing effect when integrated over the trigger angle. This behavior is in agreement with data [28]. 2) When going from central to peripheral collisions, the near-side ridge decreases and the away-side ridge changes from double to single hump, as seen in Fig. 3. This is in agreement with data [28]. 3) The correlation can also be studied as a function of the trigger-particle angle with relation to the event plane. In Fig. 4 for a mid-central window, the away-side ridge changes from single peak for in-plane trigger to double peak for out-of-plane trigger. For central collisions (not shown), it is always doublepeaked. This is in agreement with data [29]. 


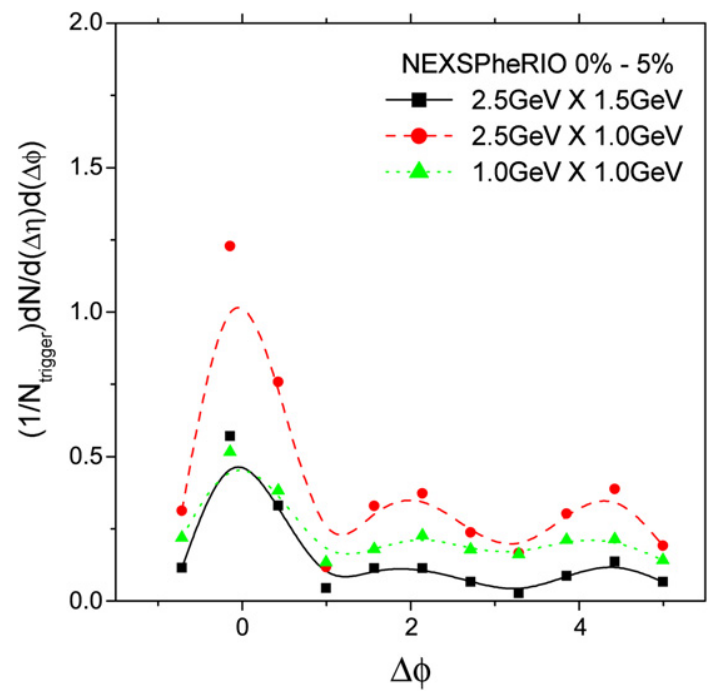

Fig. 2. $p_{t}$ dependence of NeXSPheRIO two-particle correlations for 0-20\% Au+Au collisions. Left column in legend is trigger momentum and right, associated particle momentum.
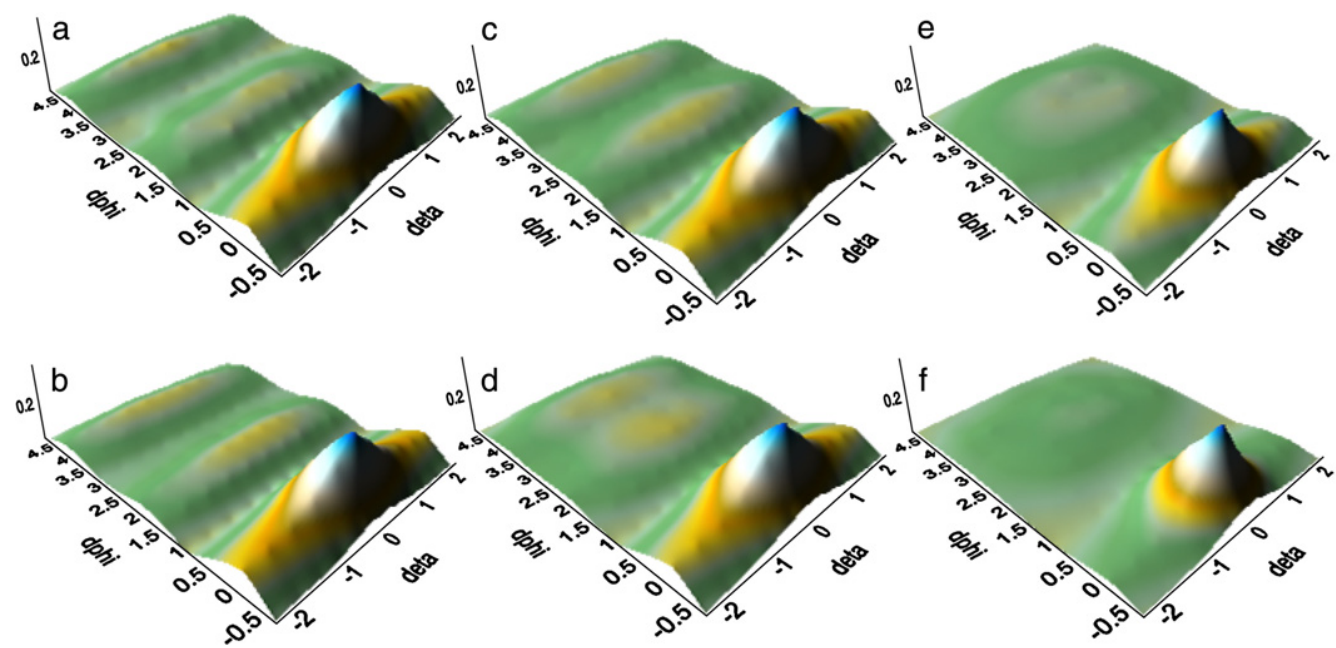

Fig. 3. NeXSPheRIO two-particle correlations for central (a) to peripheral (f) collisions.

\section{2+1 hydrodynamics: one-tube model}

When using NeXSPheRIO, it is not clear how the various structures in the two-particle correlations are generated. To investigate this, we use a simplified model. Fig. 1 (left and center) shows a typical example of initial conditions (initial energy density) obtained in NeXus with various tubular structures along the collision axis. In the simplified model, only one of the highenergy tubes from NeXus (chosen close to the border) is considered and the complex background is smoothed out by using the average over many events (for details see [25]). Transverse expansion is computed numerically while longitudinal expansion is assumed boost-invariant, until 

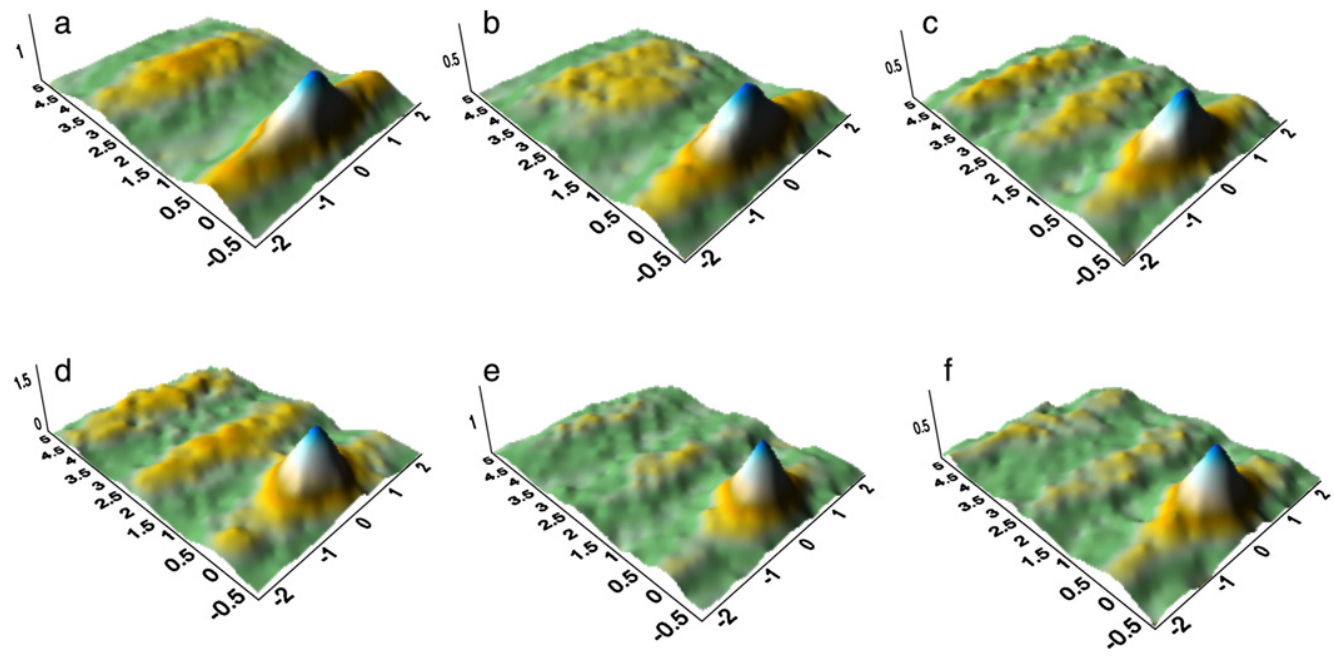

Fig. 4. NeXSPheRIO (20-30\%) results for in-plane (a) to out-of-plane (f) trigger.
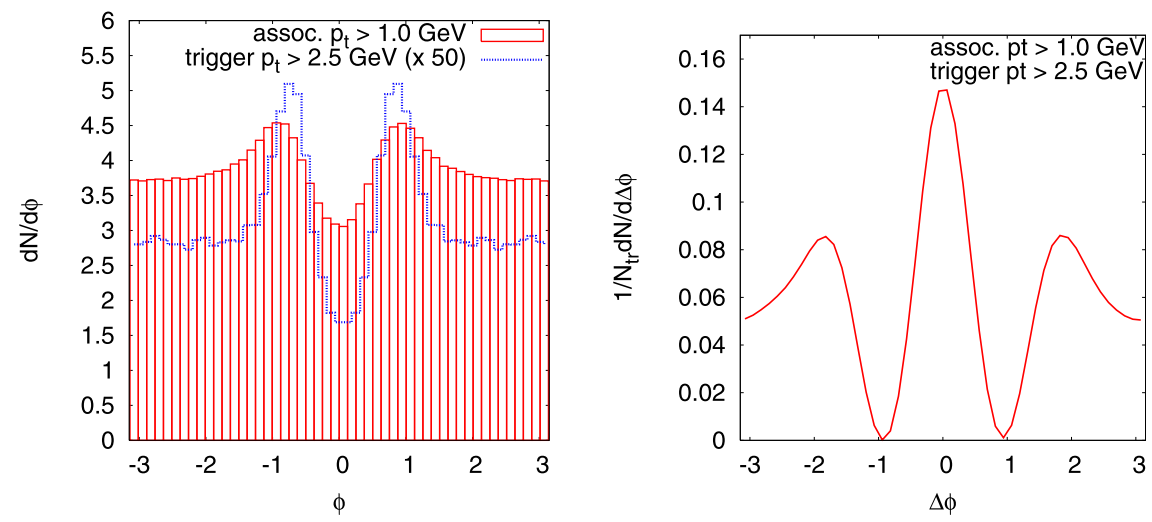

Fig. 5. Angular distributions of particles in some different $p_{T}$ intervals, in the simplified model and resulting two-particle correlations.

freeze out at some constant temperature. The resulting single-particle angular distribution has two peaks located on both sides of the position of the tube placed at $\phi=0$ as seen in Fig. 5 (left). This double peak structure is observed for all transverse momenta at more or less the same position.

This two-peak emission is in contrast with what happens when a blast wave is assumed, namely the fact that high-energy tubes emit in a single direction. However its occurrence can be understood from Fig. 6. As time goes on, as a consequence of the tube expansion, a hole appears at the location of the high-energy tube (as in [30]). This hole is surrounded by matter that piles up in a roughly semi-circular cliff of high-energy density matter, guiding the flow of the background matter into two well-defined directions. The outer part of the circle does not appear because the vacuum does not offer any resistance against the tube expansion and this part of the fluid is emitted isotropically. The two extremities of the cliff emit more fast particles than the background, this gives rise to the two-peaks in the single-particle angular distribution. 

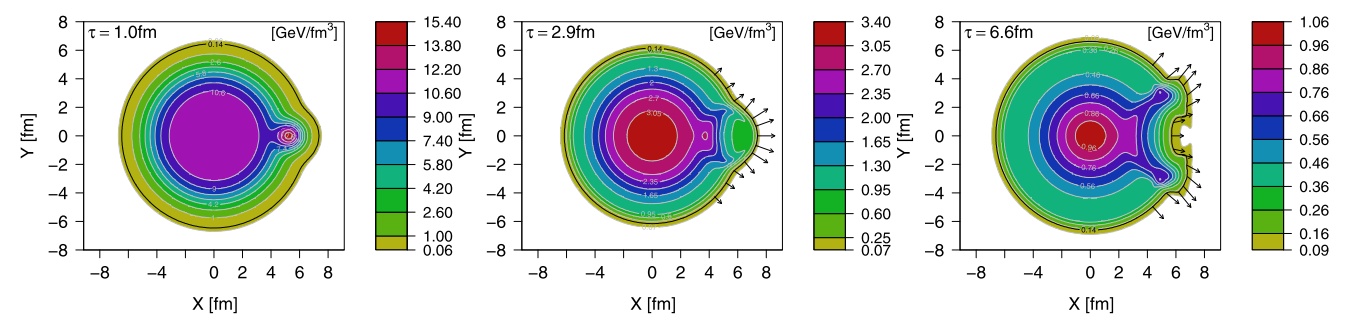

Fig. 6. Temporal evolution of energy density for the simplified model (times: 1.0, 2.9 and 6.6 fm). Arrows indicate fluid velocity on the freeze out surface, thicker curve labeled by the freeze out temperature $0.14 \mathrm{GeV}$.
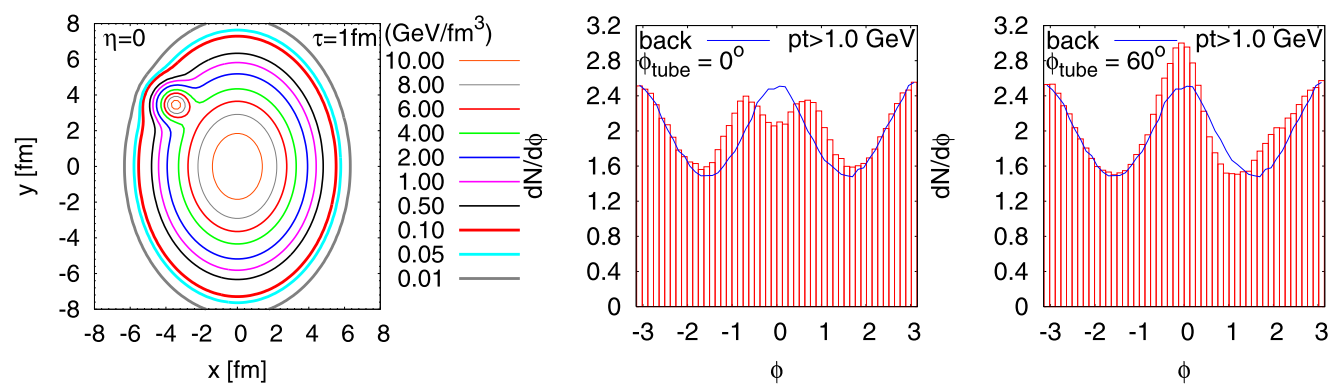

Fig. 7. Tube model for a non-central collision (20-30\% centrality). From left to right: Energy density distribution, singleparticle angular distribution for tube in the reaction plane, idem but tube at $60^{\circ}$.

The emission is not quite radial as shown by Fig. 6, indicating that there was a deflection of the background flow caused by the pressure put by the high-energy tube.

From Fig. 5 (left), we can guess how the two-particle angular correlation will be. The trigger particle is more likely to be in one of the two peaks. We first choose the left-hand side peak. The associated particle is more likely to be also in this peak, i.e. with $\Delta \phi=0$, or in the righthand side peak with $\Delta \phi \sim+2$. If we choose the trigger particle in the right-hand side peak, the associated particle is more likely to be also in this peak, i.e. with $\Delta \phi=0$, or in the left-hand side peak with $\Delta \phi \sim-2$. So the final two-particle angular correlation must have a large central peak at $\Delta \phi=0$ and two smaller peaks respectively at $\Delta \phi \sim \pm 2$. Fig. 5 (right) shows that this is indeed the case. The peak at $\Delta \phi=0$ corresponds to the near-side ridge and the peaks at $\Delta \phi \sim \pm 2$ form the double-hump ridge. We have checked that this structure is robust by studying the effect of the height and shape of the background, initial velocity, height, radius and location of the tube [25].

The results above were obtained for central collisions. We have also generalized this onetube model to non-central collisions. In Fig. 7, the single-particle angular distribution is shown with and without tube. When the tube is present, the single-particle angular distribution may also have two peaks with separation $\Delta \phi \sim 2$ or this structure may be more hidden by the elliptic flow depending on the tube position. After subtracting the elliptic flow and averaging over the tube angular position, the two-particle correlation has a near-side peak and an away-side substructure that is indeed double-peaked for more central collisions and single-peak for more peripheral ones. We have also studied the dependence on the trigger angle with respect to the reaction plane and found the correct behavior (single peak for in-plane trigger and double peak for out-of-plane one). This result is shown in Fig. 8. 


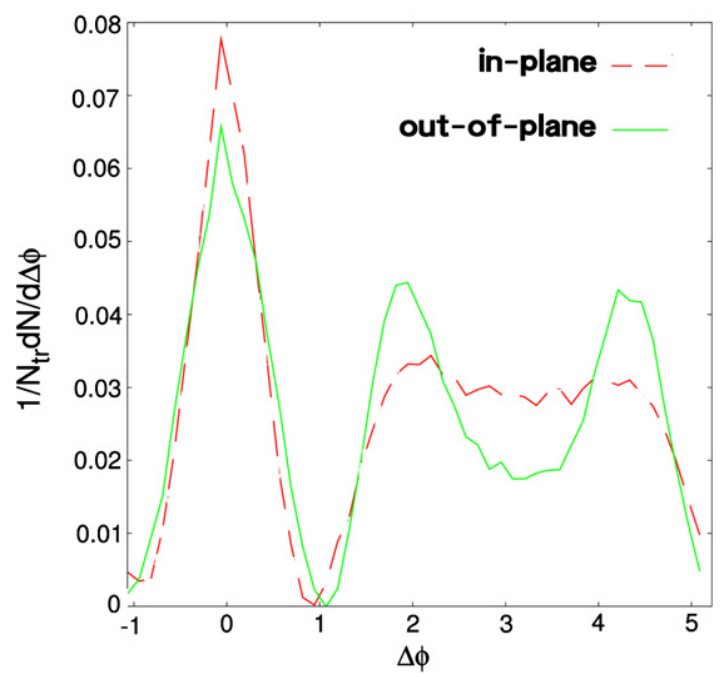

Fig. 8. Two-particle correlation for different trigger (not tube) angle.

With these information, we can discuss what happens in a more complex event such as a NeXus event. In such an event, only the outer tubes need to be considered. The shape of the twoparticle correlations for a single tube (in particular the peak spacing) is relatively independent of its features so the various tubes will contribute with rather similar two-peaks emission pattern at various angles in the single-particle angular distribution. For this single event, the two-particle correlation has a well-defined main structure similar to that of a single tube (Fig. 5) surrounded by several other peaks and depressions due to trigger and associated particles coming from different tubes. These additional peaks and depressions have positions depending on the angle of the tubes between them. When averaging over many randomly fluctuating events these interference terms disappear and only the main one-tube like structure is left.

\section{Conclusion}

To summarize, a unified picture for the structures observed in two-particle correlations at low to moderate transverse momentum was presented. It is based on the presence of longitudinal high-energy-density tubes in the initial conditions. These tubes are leftover from the initial particle interactions. During the hydrodynamical evolution of the fluid, pressed by the strong expansion of the tubes located close to the border, matter (coming mainly from the background) is deflected into two symmetrical directions, leading to two-particle correlations with a near-side and a double hump away-side ridges.

Various models have been suggested to explain the structures in the two-particle correlations and even those based on hydrodynamics have strong differences among them: for each tube, there may be no emission [30], emission in one direction [10-12] or two directions as advocated here. Three-particle correlations have been suggested as a possible test to distinguish between various scenarios. For example, three-particle correlations can be plotted as function of the angles $\Delta \phi_{1}$ between the first associated particle and the trigger and $\Delta \phi_{2}$ between the second associated particle and the trigger. The Mach cone model [31] or the AMPT model [32] would lead to off diagonal peaks in this plot while certain models might not. Experimentally, such off diagonal 

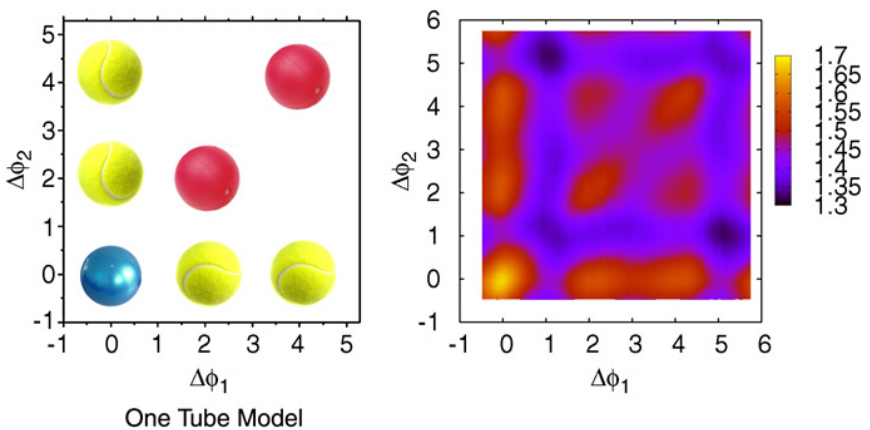

Fig. 9. Three-particle correlation in $\Delta \phi_{1}-\Delta \phi_{2}$ : naive expectation for the one-tube model and computed raw correlation.

peaks do appear in three-particle correlations [33] (see also the approach in [34]). Naively, in our case, one might expect no off diagonal peaks because there are only two peaks in the singleparticle angular distribution (Fig. 9 (left)). However if one carefully consider the contributions coming from different 3-dimensional integration domains (in $\phi_{t}, \phi_{1}$ and $\phi_{2}$ ), there appear also two off-diagonal local maxima in the three-particle correlation. This can be seen in the computed result in Fig. 9 (right). So this type of correlation does not look promising to test our model.

Another possibility would be $2+1$ correlations, where the trigger and first associated particle are chosen in different peaks of the single-particle angular distribution $\left(\Delta \phi_{1} \sim 2\right)$ and the second associated particle position varies. When this correlation is plotted as function of $\Delta \phi_{2}$ and $\Delta \eta_{2}$ (pseudorapidity difference with respect to the trigger), there should appear two main structures elongated in $\Delta \eta_{2}$ corresponding to the two possibilities for the second associated particle: it comes from the same peak as the trigger or the same peak as the first associated particle. Though it is not ruled out, it seems difficult that jet based models lead to this.

To conclude: usually, the initial conditions in the hydrodynamic description of relativistic nuclear collisions are assumed to be smooth. It seems however that each time more data require a knowledge of the event-by-event fluctuating initial conditions rather than smooth initial conditions (for example fluctuations in elliptic flow [35] or perhaps the very behavior of elliptic flow as function of pseudorapity [36]). Now two-particle correlations might also offer us a chance to get a glimpse of the initial conditions and learn about the strong interaction.

\section{Acknowledgement}

We acknowledge funding from CNPq and FAPESP.

\section{References}

[1] J. Putschke, STAR Collaboration, Nucl. Phys. A 783 (2007) 507.

[2] J. Putschke, STAR Collaboration, J. Phys. G 34 (2007) S679.

[3] M.P. McCumber, PHENIX Collaboration, J. Phys. G 35 (2007) 104081.

[4] M.J. Horner, STAR Collaboration, J. Phys. G 34 (2007) S995.

[5] E. Wenger, PHOBOS Collaboration, J. Phys. G 35 (2008) 104080.

[6] B. Alver, et al., PHOBOS Collaboration, Phys. Rev. Lett. 104 (2010) 062301.

[7] M. Daugherity, STAR Collaboration, J. Phys. G 35 (2008) 104090.

[8] S. Voloshin, Phys. Lett. B 632 (2006) 490.

[9] E. Shuryak, Phys. Rev. C 76 (2007) 047901.

[10] A. Dumitru, et al., Nucl. Phys. A 810 (2008) 91. 
[11] S. Gavin, et al., Phys. Rev. C 79 (2009) 051902.

[12] G. Moschelli, S. Gavin, arXiv:0910.3590v2.

[13] T. Hirano, K. Tsuda, Phys. Rev. C 66 (2002) 054905.

[14] C. Nonaka, S.A. Bass, Phys. Rev. C 75 (2007) 014902.

[15] C.E. Aguiar, Y. Hama, T. Kodama, T. Osada, J. Phys. G 27 (2001) 75.

[16] C.E. Aguiar, Y. Hama, T. Kodama, T. Osada, J. Phys. G 27 (2001) 551.

[17] C.E. Aguiar, Y. Hama, T. Kodama, T. Osada, Nucl. Phys. A 698 (2002) 639c.

[18] O. Socolowski Jr., F. Grassi, Y. Hama, T. Kodama, Phys. Rev. Lett. 93 (2004) 18230.

[19] R. Andrade, F. Grassi, Y. Hama, O. Socolowski Jr., T. Kodama, Phys. Rev. Lett. 97 (2006) 202302.

[20] R.P.G. Andrade, F. Grassi, Y. Hama, T. Kodama, W.-L. Qian, Phys. Rev. Lett. 101 (2008) 112301.

[21] J. Takahashi, B.M. Tavares, W.-L. Qian, R. Andrade, F. Grassi, Y. Hama, T. Kodama, N. Xu, Phys. Rev. Lett. 103 (2009) 242301.

[22] H. Petersen, J. Steinheimer, G. Burau, M. Bleicher, H. Stöcker, Phys. Rev. C 78 (2008) 044901.

[23] K. Werner, Iu. Karpenko, T. Pierog, M. Bleicher, K. Mikhailov, arXiv:1004.0805.

[24] H.J. Drescher, F.M. Liu, S. Ostapchenko, T. Pierog, K. Werner, Phys. Rev. C 65 (2002) 054902.

[25] Y. Hama, R.P.G. Andrade, F. Grassi, W.-L. Qian, Nonlinear Phenom. Complex Syst. 12 (2010) 446.

[26] R.P.G. Andrade, F. Grassi, Y. Hama, W.-L. Qian, arXiv:0912.0703.

[27] K. Werner, private communication.

[28] A. Adare, et al., PHENIX Collaboration, Phys. Rev. C 78 (2008) 014901.

[29] A. Feng, STAR Collaboration, J. Phys. G 35 (2008) 104082.

[30] E. Shuryak, Phys. Rev. C 80 (2009) 054908.

[31] T. Renk, J. Ruppert, Phys. Rev. C 76 (2007) 014908.

[32] G.L. Ma, et al., Phys. Lett. B 647 (2007) 122.

[33] B.I. Abelev, et al., STAR Collaboration, Phys. Rev. Lett. 102 (2009) 052302.

[34] N.N. Ajitanand, PHENIX Collaboration, Nucl. Phys. A 783 (2007) 519.

[35] Y. Hama, R.P.G. Andrade, F. Grassi, W.-L. Qian, T. Osada, C.E. Aguiar, T. Kodama, Phys. Atom. Nucl. 71 (2008) 1558.

[36] R.P.G. Andrade, F. Grassi, Y. Hama, T. Kodama, W.-L. Qian, Phys. Rev. Lett. 101 (2008) 112301. 